\title{
On the Stiffness Affected by the Allocation of Actuators of a Parallel Manipulator
}

\author{
Wanghui $\mathrm{Bu}^{*}$
}

School of Mechanical Engineering, Tongji University, Shanghai 200092, China

\begin{abstract}
The stiffness of a parallel manipulator is not only concerned with its detailed structure but also affected by the allocation scheme of actuators. Even though the precise values of stiffness cannot be derived since the link structure is not definitive at the stage of mechanism design, the effect of the allocation of actuators on the mechanism stiffness can be qualitatively evaluated in order to guild the optimal allocation. In this paper, the mechanism stiffness of a parallel manipulator is classified into the actuated stiffness and the structural stiffness. The actuated stiffness is obtained through the principle of virtual displacement and Jacobian matrix, and it is presented as a stiffness matrix which contains all the stiffness components and maps the stiffness of each actuator into the stiffness of the moving platform. The proposed analysis method of the actuated stiffness is suitable for all types of actuators. The structural stiffness is analyzed based on the principle of virtual force. A parallel mechanism is converted into a stationary structure when all active joints are frozen. The stationary structure is regarded as the combination of the basic links which are categorized according to their geometric shapes, join types, and load conditions. The proposed analysis method of structural stiffness takes account of all kinds of internal forces such as axial force, shearing force, bending moment, and torque. It can be seen that the allocation scheme of actuators plays an important role in the contribution to the stiffness of a parallel mechanism. The stiffness analysis that is usually investigated at the stage of structure design in the traditional process of mechanical design is considered in anticipation at the stage of mechanism design in the proposed approach. The stiffness of the moving platform affected by the allocation of actuators is analyzed through the simplified mechanism model without the detailed geometric shapes or physical parameters of link parts, which is able to guild the optimal selection of an allocation scheme of actuators at the early stage of the design process.
\end{abstract}

Keywords: Parallel robots, actuation, mechanism stiffness, principle of virtual work.

\section{INTRODUCTION}

Parallel manipulators have been applied more and more in industry due to their high stiffness, although their workspaces are confined compared with the serial manipulators. The stiffness of the moving platform of a parallel manipulator can be classified into two types: one is called the actuated stiffness which represents the effect of the stiffness of each actuator; the other is called the structural stiffness which represents the effect of each component structure of the manipulator. Generally, the mechanism stiffness is expected to be improved as high as possible in the limitation of the total weight and manufacturing cost, which requires that the stiffness is not only implemented at the stage of structure design but also considered sufficiently at the stage of mechanism design.

Many researchers have studied the mechanism stiffness of parallel manipulators. Carbone and Ceccarelli [1] evaluated the stiffness of a hybrid parallel-serial manipulator, and the axial stiffness of each link and radial stiffness of each joint were taken into account while the flexion deformations of links were ignored. In their work, the stiffness matrix that was deduced as a function of the most important stiffness and design parameters was achieved and utilized to assess

*Address correspondence to this author at the School of Mechanical Engineering, Tongji University, Shanghai 200092, China; Tel: +86 139 8987 1476; E-mail: buwanghui@gmail.com the stiffness performance. They [2] also compared different indices to evaluate the local and global stiffness performances of robotic manipulators, and proposed new global stiffness indices based on the integration of the local stiffness indices. Yoon et al., [3] analyzed the stiffness of a compact modified Delta parallel mechanism with the consideration of the elastic deformations of both links and bearings. They derived the compliance matrix of this mechanism, and obtained the elastic coefficient value of the rotation axis of the bearing. $\mathrm{Xu}$ and $\mathrm{Li} \mathrm{[4]} \mathrm{investigated} \mathrm{on} \mathrm{the}$ mobility and stiffness of a 3-DOF translational parallel manipulator through the screw theory. In their work, the original 3-PRC mechanism was converted into a non-overconstrained 3-CRC mechanism, and then the stiffness matrix was obtained with the consideration of the compliances of both actuators and links, finally the stiffness performance was evaluated using the extreme values of stiffness over the reachable workspace. Bonnemains et al., [5] researched the stiffness computation and identification of parallel machine tools with the consideration of the compliances of both links and joints. They modeled the links as beams, and the stiffness model of each joint was established through the local behavior of the joint elements. Lu et al., [6] analyzed the stiffness and elastic deformation of a 2(3-SPR) serialparallel manipulator. They gained the compliance matrix of each link and obtained the total stiffness matrix and the elastic deformation based on the principle of virtual work. Pashkevich et al., [7] proposed an approach of stiffness modeling for overconstrained parallel manipulators with 
flexible links and compliant actuators. They presented a multidimensional lumped-parameter model and localized 6-DOF virtual springs to describe the translational and rotational relative motion between the links, and gained the stiffness matrix from a finite element model. Wu et al., [8] studied the stiffness of a 5-DOF hybrid machine tool with redundant actuation. The stiffness of each subsystem such as the revolute pair and the guideway slider was derived through the experimental measurement, and was assembled into an entire stiffness model of the mechanism. They also proposed some stiffness evaluation indices to compare the stiffness between the redundant and non-redundant machine tools. Enferadi and Tootoonchi [9] adopted the strain energy and Castigliano's theorem to analyze the structural stiffness of a 3-RRP spherical parallel manipulator, and used a finite element model to verify the theoretical analysis. The flexibility of the moving platform was taken into consideration while the actuated stiffness was neglected. Gao et al., [10] studied the stiffness analysis and optimization of a 3-DOF parallel manipulator based on the Jacobian matrix. In their work, the actuated stiffness was considered while the structural stiffness was neglected. The mean value and the standard deviation of the global stiffness distribution were proposed as the design indices, and the genetic algorithm and artificial neural networks were utilized for the stiffness optimization. Pinto et al., [11] established an elastic model and a finite element-based model of a parallel manipulator with lower mobility and decoupled motion, and parameters of these models were adjusted and verified through an experimental prototype, and in this way the stiffness mapping from each component structure to the moving platform was obtained. In their work, the structural stiffness was considered while the actuated stiffness was ignored. Vaidya and Padole [12] modeled the joint in a four-bar mechanism with the help of a clearance link that was assigned with proper axial, longitudinal and torsional stiffness. They observed that as the joint stiffness increased, the natural frequency of the four-bar mechanism also increased and converged.

It is noted that, although there are many researches on the mechanism stiffness, the investigation on the relation between the mechanism stiffness and the allocation schemes of actuators has hardly been studied. In fact, the stiffness of a parallel manipulator is not only concerned with the detailed structure, but also affected by the allocation of actuators. A planar 3-DOF serial manipulator is shown in Fig. (1), and the actuators are allocated at joints $J_{1}, J_{2}$ and $J_{3}$; a planar 3-DOF

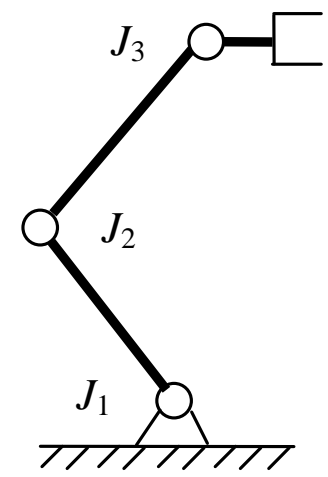

Fig. (1). A planar3-DOF serial manipulator.

parallel manipulator is shown in Fig. (2), and the three actuators are also allocated at joints $J_{1}, J_{2}$ and $J_{3}$. Comparing these two mechanisms, we can make a judgment by intuition that the other limb where no actuator is allocated cannot give any support to the moving platform of the parallel manipulator; on the contrary, if the gravity of the other limb is taken into account, the stiffness of the moving platform of the parallel manipulator is less than the stiffness of the endeffector of the serial one. Hence, it can be seen that the allocation scheme of actuators indeed affects the stiffness of parallel mechanisms. In this paper, both actuated stiffness and structural stiffness affected by different allocation schemes of actuators are investigated using the principles of virtual displacement and virtual force.

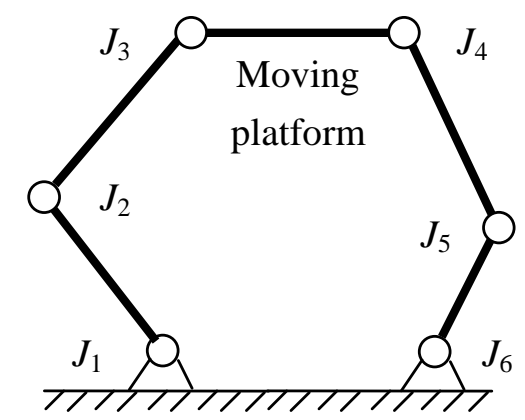

Fig. (2). A planar 3-DOF parallel manipulator.

It is also worth notice that, the stiffness should be considered at the stage of mechanism design, but the precise value of the stiffness cannot be derived since the link structure is not definitive. However, the effect of the allocation of actuators on the mechanism stiffness can be qualitatively evaluated in order to guild the optimal allocation. In this paper, the links are classified into 36 types of basic links based on their sketch shapes and external loads so as to estimate the structural stiffness.

The remaining of this paper is organized as follows. In Section 2, the actuated stiffness is analyzed based on the principle of virtual displacement. In Section 3, the structural stiffness is calculated based on the principle of virtual force. In Section 4, the total stiffness of a parallel mechanism and the optimal allocation of actuators are studied. A case of a planar 3-DOF parallel manipulator is investigated and discussed in Section 5. Finally, conclusions are given in Section 6 .

\section{ANALYSIS OF ACTUATED STIFFNESS BASED ON THE PRINCIPLE OF VIRTUAL DISPLACEMENT}

The active joints of a parallel manipulator are mounted with actuators which control the moving platform to travel along the desired trajectory. When these actuators are frozen, the mechanism cannot move any more. However, an actual actuator must have some compliance that causes the actuator to generate a slight elastic deformation along the driving direction under an external load. If the flexibility of links is ignored and only the compliance of actuators is taken into account, the ratio of the external load at the moving platform to the slight displacement caused by the external load is defined as the actuated stiffness of a parallel mechanism. The actuated stiffness depends on the pose of the mechanism; for a special pose, when the compliance of actuators is in linear elastic scope, the actuated stiffness is a natural 
property of the mechanism, which means the actuated stiffness is invariable and is independent of the external load. Note that the actuated stiffness has the directional property, that is to say, the external loads with identical magnitude along different directions may generate the slight displacements of the moving platform with different magnitudes. Fig. (3) shows the displacement of the moving platform caused by the actuated stiffness, where the actuators are mounted at joints $J_{1}, J_{2}$ and $J_{6}$. The dashed and real lines in Fig. (3) denote the original position before deformation and the real position after deformation, respectively. Note that the slight deformation is magnified for clear presentation in Fig. (3).

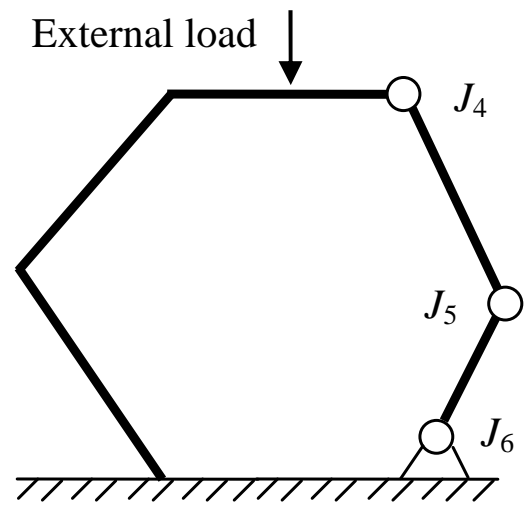

Fig. (3). Displacement of the moving platform caused by actuated stiffness.

Let $F_{M P}$ be the external load, $K_{A}$ the actuated stiffness of the moving platform, $\Delta_{A}$ the elastic deformation of the moving platform brought by the actuator compliance, so according to the Hooke's law, we obtain

$F_{M P}=K_{A} \cdot \Delta_{A}$

For each active joint, the following equation exists when the elastic deformation is taken into consideration

$f_{i}=k_{i} \cdot \delta_{i}$

where $f_{i}$ denotes the external load component along the driving direction, $k_{i}$ the stiffness of the actuator, $\delta_{i}$ the slight elastic deformation of the actuator along the driving direction. If there are $I$ actuators mounted in the mechanism, the following matrix equation exists

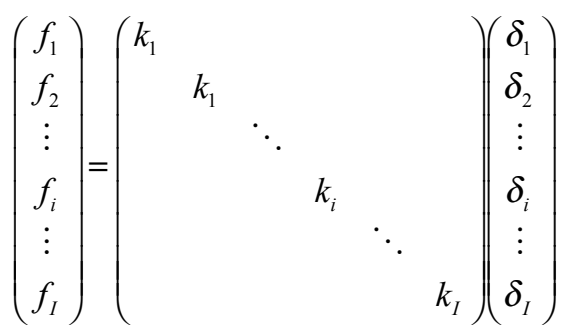

Each matrix in the above equation can be represented as a symbol, and the equation can be rewritten as

$F_{I}=K_{I} \cdot \Delta_{I}$

Now we do not consider other elastic deformations such as the structural flexibility, and $\Delta_{A}$ and $\Delta_{I}$ are regarded as the virtual displacements of the moving platform and the actuators, respectively. Therefore according to the principle of virtual displacement, there exists

$F_{M P}^{T} \cdot \Delta_{A}=F_{I}^{T} \cdot \Delta_{I}$

Substituting Eqns. (1) and (4) into Eqn. (5) gives the following equation

$\Delta_{A}^{T} \cdot K_{A}^{T} \cdot \Delta_{A}=\Delta_{I}^{T} \cdot K_{I}^{T} \cdot \Delta_{I}$

The Jacobian matrix of a mechanism describes the velocity mapping from the joints to the moving platform. The virtual displacements can be regarded as the instantaneous velocities, hence we obtain

$\Delta_{A}=J \cdot \Delta_{I}$

Substituting Eqn. (7) into Eqn. (6) yields

$\Delta_{I}^{T} \cdot J^{T} \cdot K_{A}^{T} \cdot J \cdot \Delta_{I}=\Delta_{I}^{T} \cdot K_{I}^{T} \cdot \Delta_{I}$

Thereby the actuated stiffness of the moving platform has been obtained as the function of the stiffness of each actuator

$K_{A}=\left(\Delta_{I}^{T} \cdot J^{T}\right)^{-1} \cdot \Delta_{I}^{T} \cdot K_{I} \cdot \Delta_{I} \cdot\left(J \cdot \Delta_{I}\right)^{-1}$

When the inverse of the Jacobian matrix exists, Eqn. (9) is simplified as

$$
K_{A}=\left(J^{-1}\right)^{T} \cdot K_{I} \cdot J^{-1}
$$

It is noted that, some research works did not identify the existence of the inverse of the Jacobian matrix, which were incorrect to use Eqn. (10) to calculate the actuated stiffness. For a manipulator with lower mobility, the Jacobian matrix is not the square matrix, so its inverse matrix does not exist, which indicates that the actuated stiffness has to be calculated from Eqn. (9).

\section{ANALYSIS OF THE STRUCTURAL STIFFNESS BASED ON THE PRINCIPLE OF VIRTUAL FORCE}

When all active joints are frozen, a moveable mechanism is converted into a stationary structure. If there are different schemes for the allocation of actuators, the same mechanism will turn into many different structures. For the planar 3DOF parallel manipulator as shown in Fig. (2), if the actuators are mounted at joints $J_{1}, J_{2}$ and $J_{3}$, the converted structure after freeing three actuators is shown in Fig. (4); if the actuators are mounted at joints $J_{1}, J_{2}$ and $J_{6}$, the converted structure after freeing three actuators is shown in Fig. (5).

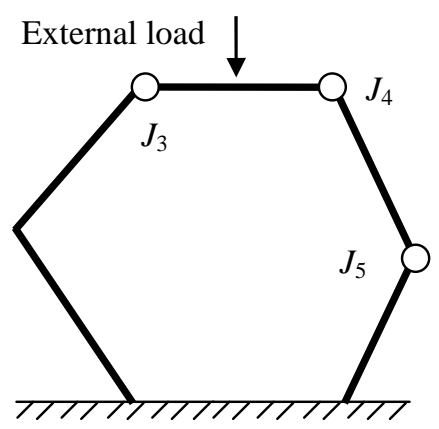

Fig. (4). The structure converted from the parallel manipulator when $J_{1}, J_{2}$ and $J_{3}$ are frozen. 


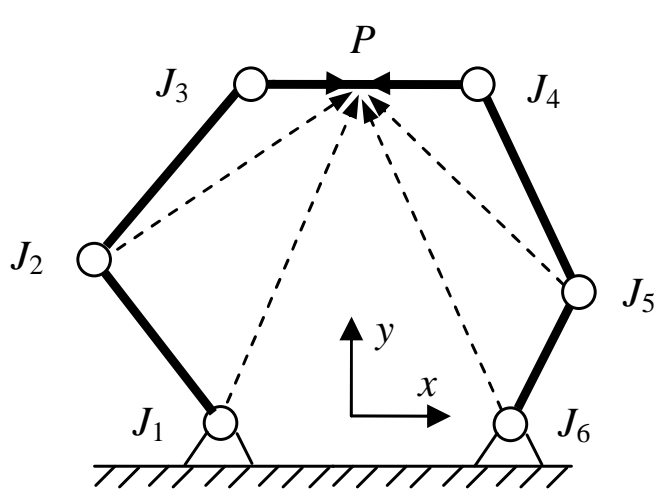

Fig. (5). The structure converted from the parallel manipulator when $J_{1}, J_{2}$ and $J_{6}$ are frozen.

The actual links of a manipulator must have certain flexibilities which cause the slight elastic deformations of links under an external load. Hence, it is necessary to analyze the effect of the stiffness of each link on the stiffness of the moving platform. If the compliance of actuators is ignored and only the flexibility of links is taken into account, the ratio of the external load at the moving platform to the slight displacement caused by the external load is defined as the structural stiffness of a parallel mechanism. The structural stiffness depends on the pose of the mechanism; for a special pose, when the flexibility of links is in linear elastic scope, the structural stiffness is a natural property of the mechanism, which means the structural stiffness is invariable and is independent of the external load. Note that the structural stiffness has the directional property, that is to say, the external loads with identical magnitude along different directions may generate the slight displacements of the moving platform with different magnitudes. Since a parallel manipulator can be converted into many different structures due to the different allocation schemes of actuators, the structural stiffness is indeed affected by the allocation schemes. Fig. (6) shows the displacement of the moving platform caused by the structural stiffness, where the actuators are mounted at joints $J_{1}, J_{2}$ and $J_{6}$. The dashed and real lines in Fig. (6) denote the original position before deformation and the real position after deformation, respectively. Note that the slight deformation is magnified for clear presentation in Fig. (6).

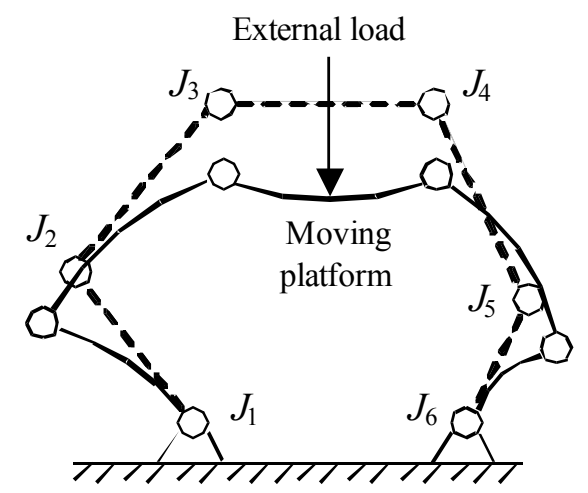

Fig. (6). Displacement of the moving platform caused by structural stiffness.

When we calculate the stiffness of a structure, we need to know the geometric shape and the physical characteristics of the structure. But for a parallel manipulator at the stage of mechanism design, we achieve only the simplified model where the dimensions of links are given but the detailed structural parameters are not known. Fortunately, we just analyze the effect of the allocation schemes of actuators on the mechanism stiffness of the simplified model at the stage of mechanism design, so we do not care for the precise values of the structural stiffness. Hence, in this paper the components of a parallel manipulator are assumed as the simplified links which have the same Young's modulus and cross-sectional shape. Besides, when we deal with the structural stiffness, the elastic deformations of actuators are ignored, and the final mechanism stiffness will be the linear superimposition of the structural stiffness and the actuated stiffness.

In order to obtain the stiffness of each link, components are classified into a series of basic links according to their geometric shapes, join types, and load conditions. As for planar mechanisms, there are two kinds of geometric shapes that are straight links and fractional links; three join types that are revolute joints, prismatic joints, and fixed joints; two kinds of load conditions that are with or without the intermediate external loads. Since a link should be joined at two ends, there are $N=2 \times 3^{2} \times 2=36$ types of the basic links as shown in Table $\mathbf{1}$. The joint types I and II in Table $\mathbf{1}$ denote the types of two joints at two ends of each link, and the number of these two types is the same.

For a planar parallel manipulator without redundant actuation, the mechanism can be converted into a statically determinate structure whose joint reaction forces can be solved from the external load $F_{M P}$ at the moving platform according to the static equilibrium. Then all internal forces of each link such as the axial force $F_{N}$, shearing force $F_{Q}$, bending moment $M$, and torque $M_{t}$ are worked out. All links are assumed to be made of linear elastic material, so the strains generated by the internal forces are

$$
\begin{gathered}
\varepsilon=\frac{F_{N}}{E A} \\
\gamma=\frac{k F_{Q}}{G A} \\
\kappa=\frac{M}{E I_{z}} \\
\varphi^{\prime}=\frac{M_{t}}{G I_{t}}
\end{gathered}
$$

where $\varepsilon$ denotes the axial strain, $\gamma$ the shearing strain, $\kappa$ the curvature of the link axis, $\varphi^{\prime}$ the relative rotation of two cross sections along the unit length, $E$ the Young's modulus, $G$ the shearing modulus, $A$ the area of the cross section, $I_{z}$ the moment of inertia, $I_{t}$ the polar moment of inertia.

Let $\Delta_{S}$ be the slight elastic deformation of the moving platform caused by the structural flexibility. It is assumed that a unit external load 1 is added at the moving platform, and then the virtual work applied by the unit load 1 along the direction of $\Delta_{S}$ is

$W_{e}=1 \cdot \Delta_{S}=\Delta_{S}$ 
Table 1. Types of the Basic Links

\begin{tabular}{|c|c|c|c|}
\hline Geometric Shapes & Join Types I & Join Types II & Load Conditions \\
\hline (a) A Straight link & (a) A revolute joint & a) A revolute joint & (a) Without intermediate load \\
\hline (b) A fractional link & (b) A prismatic joint & (b) A prismatic joint & $\begin{array}{l}\text { Evernal load } \\
\text { (b) With intermediate loads }\end{array}$ \\
\hline & (c) A fixed joint & (c) A fixed joint & \\
\hline
\end{tabular}

The internal forces of each link such as the axial force $\tilde{F}_{N}$, shearing force $\tilde{F}_{Q}$, bending moment $\tilde{M}$, and torque $\tilde{M}_{t}$ generated by the unit load 1 are calculated based on the static equilibrium. Note that $\tilde{F}_{N}$ and $\tilde{F}_{Q}$ are dimensionless, and the unit of $\tilde{M}$ and $\tilde{M}_{t}$ is meter. Thereby the virtual work applied by all the internal forces of all links is obtained according to the principle of superposition

$W_{i}=\sum \int_{0}^{l}\left(\frac{F_{N} \tilde{F}_{N}}{E A}+\frac{k F_{Q} \tilde{F}_{Q}}{G A}+\frac{M \tilde{M}}{E I_{z}}+\frac{M_{t} \tilde{M}_{t}}{G I_{t}}\right) d s$

where the summation symbol means the accumulation of each virtual work applied by the internal forces of each link. Note that a fractional link should be decomposed into several straight links for the calculation of its virtual work as shown in Fig. (7).

According to the principle of virtual force, there exists $W_{e}=W_{i}$. Let $K_{S}$ be the structural stiffness of the moving platform, we obtain

$$
K_{S}=\frac{F_{M P}}{\sum \int_{0}^{l}\left(\frac{F_{N} \tilde{F}_{N}}{E A}+\frac{k F_{Q} \tilde{F}_{Q}}{G A}+\frac{M \tilde{M}}{E I_{z}}+\frac{M_{t} \tilde{M}_{t}}{G I_{t}}\right) d s}
$$

The geometric and physical parameters of the basic links, such as the Young's modulus and cross-sectional areas, are assumed to be the same. If the cross section of the link is circular, the radius and length are denoted by $r$ and $L$. When the external load $F$ stretches or compresses the link at its two ends, the deformation is

$$
\delta_{1}=\frac{F L}{\pi r^{2} E}
$$

When one end of the link is fixed and the other end suffers the transversal force $F$ that bends the link, the deformation is

$$
\begin{aligned}
& \delta_{2}=\frac{4 F L^{3}}{3 \pi r^{4} E} \\
& \frac{\delta_{2}}{\delta_{1}}=\frac{4}{3}\left(\frac{L}{r}\right)^{2}
\end{aligned}
$$

When $L>2.74 r$, the bending deformation is more than 10 times as the tension or compression deformation under the external forces with the identical magnitude. When $r=$ $0.002 \mathrm{~m}$ and $L=0.5 \mathrm{~m}$, the bending deformation is 833 times as the tension or compression deformation. Hence for the planar parallel manipulators, the bending deformation is regarded as the main factor to the structural flexibility. When the secondary deformations are ignored, the structural stiffness of a parallel manipulator is simplified as

$$
K_{S}=\frac{F_{M P}}{\sum \int_{0}^{l} \frac{M \tilde{M}}{E I_{z}} d s}=\frac{E I_{z}}{\sum \int_{0}^{l} \tilde{M}^{2} d s}
$$

\section{THE TOTAL STIFFNESS AND THE OPTIMAL ALLOCATION OF ACTUATORS}

The mechanism stiffness $K$, total deformation of the moving platform $\Delta$, and the external load $F_{M P}$ should satisfy the Hooke's law

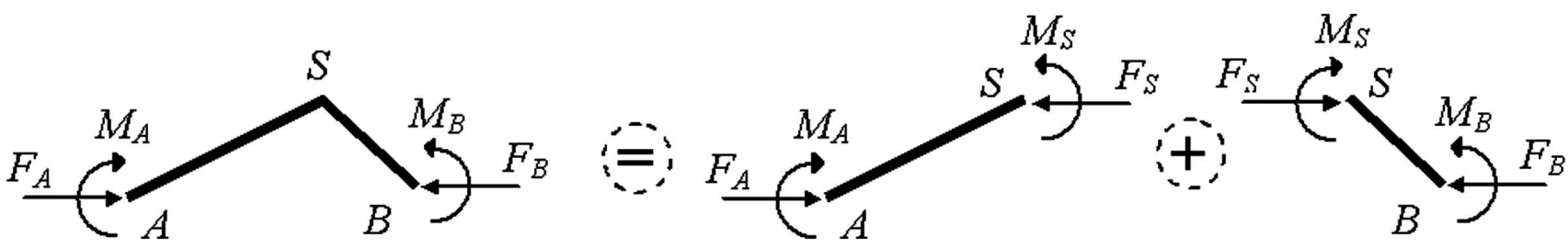

Fig. (7). The sectional solution of the fractional link. 
$F_{M P}=K \cdot \Delta$

The deformations caused by the actuated stiffness and the structural stiffness satisfy the following two equations

$\Delta_{A}=\frac{F_{M P}}{K_{A}}$

$\Delta_{S}=\frac{F_{M P}}{K_{S}}$

And the total deformation equals to the linear superimposition of the deformations caused by the two types of stiffness

$\Delta=\Delta_{A}+\Delta_{S}$

According to Eqns. (15)-(18), the total stiffness of a parallel manipulator is

$K=\frac{K_{A} K_{S}}{K_{A}+K_{S}}$

There are many design indices to be considered at the stage of mechanism design, yet some of these indices are contradictory to each other, therefore it needs to make a compromise between the design indices. Usually the mechanism stiffness is expected to be improved as high as possible, but this stiffness index is conflict with other indices such as the machine weight and manufacturing cost. Hence, the mechanism stiffness in the robotic reachable workspace is often required no less than a minimum value.

Let the numbers of total joints and active joints of a parallel manipulator be $N_{\text {all }}$ and $N_{\text {dof }}$ respectively, and then the number of allocation schemes of actuators is $N_{s c h}$

$N_{s c h}=\left(\begin{array}{l}N_{\text {all }} \\ N_{\text {dof }}\end{array}\right)=\frac{N_{\text {all }}\left(N_{\text {all }}-1\right) \cdots\left(N_{\text {all }}-N_{\text {dof }}+1\right)}{N_{\text {dof }}\left(N_{\text {dof }}-1\right) \cdots \times 2 \times 1}$

Note that the above equation does not involve the possible symmetry of the limbs. For a manipulator with symmetrical limbs, the calculation of the allocation types may be referred to the research work of Zhao et al., [13] who studied the symmetrical characteristics of workspace for a mechanism with symmetric structure.

For each allocation scheme, a running curve can be obtained according to Eqn. (19) to describe the stiffness of a parallel manipulator moving in its reachable workspace. If there is a zone of the stiffness curve below the minimum value, this allocation scheme should be abandoned. If the whole stiffness curve is above the minimum value, an optimal allocation scheme can be achieved with the comprehensive consideration of other design indices.

\section{AN EXAMPLE}

A planar 3-DOF parallel manipulator is shown in Fig. (8), and we will calculate the total mechanism stiffness affected by the allocation of actuators. According to Eqn. (20), there are $(6 \times 5 \times 4) /(3 \times 2)=20$ schemes of allocation of actuators. For the convenient discussion, only two schemes of allocation are analyzed in this paper. In one scheme, the three actuators are allocated at joints $J_{1}, J_{2}$ and $J_{3}$. In the other scheme, the three actuators are allocated at joints $J_{1}, J_{2}$ and $J_{6}$. The lengths of links of the parallel manipulator are shown in Table 2

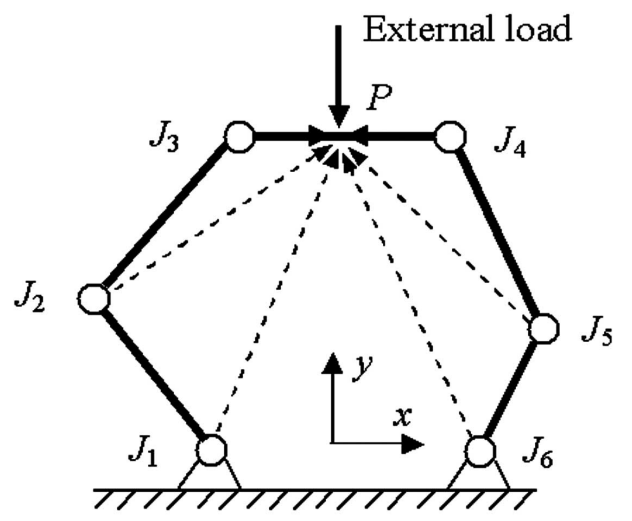

Fig. (8). A planar 3-DOF parallel manipulator and its joint vectors to construct the Jacobian matrix.

Table 2. Lengths of Links of the Planar 3-DOF Parallel Manipulator

\begin{tabular}{|c|c|c|c|}
\hline Link & Length (m) & Link & Length (m) \\
\hline \hline$J_{1} J_{2}$ & 0.9 & $J_{4} J_{5}$ & 1.0 \\
\hline$J_{2} J_{3}$ & 1.0 & $J_{5} J_{6}$ & 0.6 \\
\hline$J_{3} J_{4}$ & 0.8 & $J_{1} J_{6}$ & 1.0 \\
\hline$J_{3} P$ & 0.4 & $J_{4} P$ & 0.4 \\
\hline
\end{tabular}

Let the point $P$ of the moving platform translate along the line $P_{1} P_{5}$, and keep link $J_{3} J_{4}$ horizontal during the motion. The coordinates of the five points in line $P_{1} P_{5}$ are $P_{1}(0,1.1)$, $P_{2}(0,1.2), P_{3}(0,1.3), P_{4}(0,1.4)$, and $P_{5}(0,1.5)$. Let the stiffness of the three actuators be the same as $k_{a c t}$. The analysis process of the actuated stiffness and structural stiffness is shown in Fig. (9).

Firstly, the actuated stiffness is calculated, which needs to know the Jacobian matrix of the parallel manipulator. The kinematic model is established, and the joint angles are solved through the inverse displacement analysis according to the pose of the moving platform. The parallel mechanism can be regarded as the combination of two limbs whose junction is point $P$ in Fig. (8). The velocity of the moving platform in the left limb is affected by the velocities of joints $J_{1}, J_{2}$ and $J_{3}$

$\left(\begin{array}{c}\omega_{P} \\ v_{P x} \\ v_{P y}\end{array}\right)=\left(\begin{array}{ccc}1 & 1 & 1 \\ -\left(J_{1} P\right)_{y} & -\left(J_{2} P\right)_{y} & -\left(J_{3} P\right)_{y} \\ \left(J_{1} P\right)_{x} & \left(J_{2} P\right)_{x} & \left(J_{3} P\right)_{x}\end{array}\right)\left(\begin{array}{l}\omega_{J_{1}} \\ \omega_{J_{2}} \\ \omega_{J_{3}}\end{array}\right)$

where $\omega_{J_{1}}, \omega_{J_{2}}$, and $\omega_{J_{3}}$ are the angular velocities of joints $J_{1}, J_{2}$ and $J_{3}$, respectively; the subscripts $x$ and $y$ denote the vector components along the axes $x$ and $y$ of the reference frame.

The velocity of the moving platform in the right limb is affected by the velocities of joints $J_{1}, J_{2}$ and $J_{6}$ 


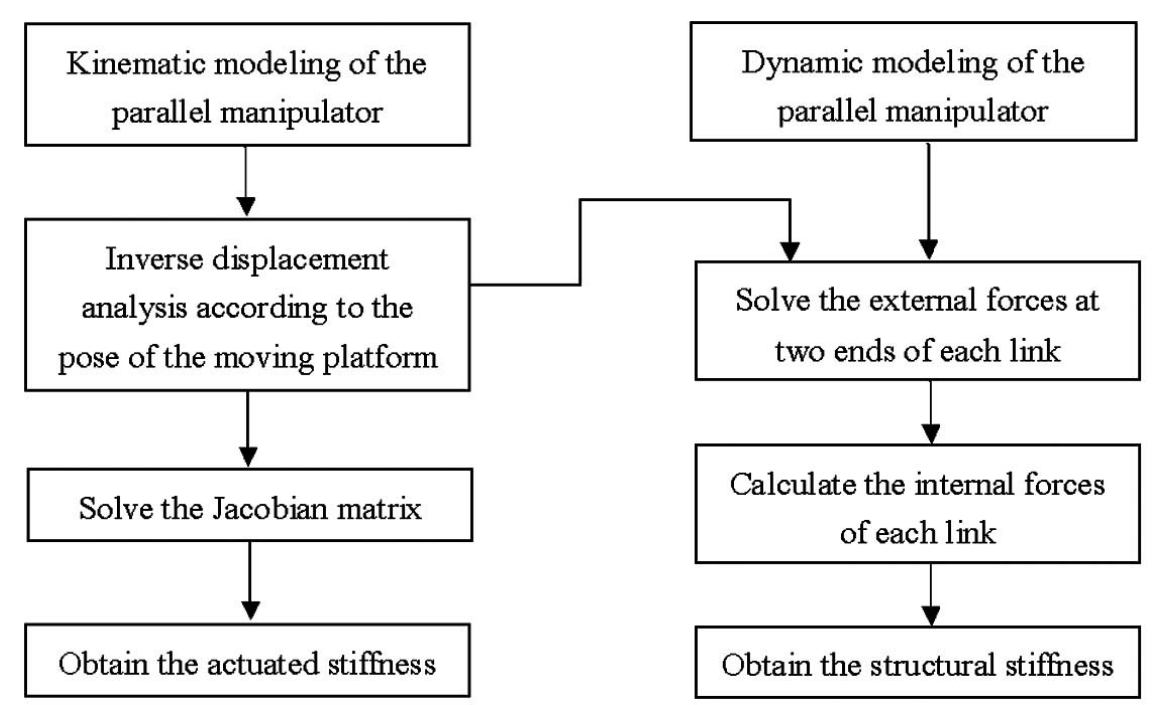

Fig. (9). Analysis process of the actuated stiffness and structural stiffness.

$$
\left(\begin{array}{c}
\omega_{P} \\
v_{P x} \\
v_{P y}
\end{array}\right)=\left(\begin{array}{ccc}
1 & 1 & 1 \\
-\left(J_{4} P\right)_{y} & -\left(J_{5} P\right)_{y} & -\left(J_{6} P\right)_{y} \\
\left(J_{4} P\right)_{x} & \left(J_{5} P\right)_{x} & \left(J_{6} P\right)_{x}
\end{array}\right)\left(\begin{array}{c}
\omega_{J_{4}} \\
\omega_{J_{5}} \\
\omega_{J_{6}}
\end{array}\right)
$$

When joints $J_{1}, J_{2}$ and $J_{3}$ are taken as the active joints, the Jacobian matrix is just the square matrix in Eqn. (21)

$$
J_{123}=\left(\begin{array}{ccc}
1 & 1 & 1 \\
-\left(J_{1} P\right)_{y} & -\left(J_{2} P\right)_{y} & -\left(J_{3} P\right)_{y} \\
\left(J_{1} P\right)_{x} & \left(J_{2} P\right)_{x} & \left(J_{3} P\right)_{x}
\end{array}\right)
$$

Hence, the Jacobian matrices of the manipulator at the five points along the line $P_{1} P_{5}$ are

$$
\begin{aligned}
& J_{123 P 1}=\left(\begin{array}{ccc}
1 & 1 & 1 \\
-1.1 & -0.566 & 0 \\
0.5 & 1.224 & 0.4
\end{array}\right), \\
& J_{123 P 2}=\left(\begin{array}{ccc}
1 & 1 & 1 \\
-1.2 & -0.618 & 0 \\
0.5 & 1.186 & 0.4
\end{array}\right), \\
& J_{123 P 3}=\left(\begin{array}{ccc}
1 & 1 & 1 \\
-1.3 & -0.669 & 0 \\
0.5 & 1.143 & 0.4
\end{array}\right), \\
& J_{123 P 4}=\left(\begin{array}{ccc}
1 & 1 & 1 \\
-1.4 & -0.722 & 0 \\
0.5 & 1.092 & 0.4
\end{array}\right), \\
& J_{123 P 5}=\left(\begin{array}{ccc}
1 & 1 & 1 \\
-1.5 & -0.774 & 0 \\
0.5 & 1.033 & 0.4
\end{array}\right),
\end{aligned}
$$

When joints $J_{1}, J_{2}$ and $J_{3}$ are taken as the active joints, the matrices of the actuated stiffness at the five points are solved according to Eqn. (10)

$$
\begin{aligned}
& K_{A 123 P 1}=k_{a c t}\left(\begin{array}{ccc}
1.904 & 0.747 & -1.632 \\
0.747 & 1.680 & 0.262 \\
-1.632 & 0.262 & 2.511
\end{array}\right), \\
& K_{A 123 P 2}=k_{a c t}\left(\begin{array}{ccc}
1.973 & 0.672 & -1.772 \\
0.672 & 1.414 & 0.265 \\
-1.772 & 0.265 & 2.779
\end{array}\right), \\
& K_{A 123 P 3}=k_{a c t}\left(\begin{array}{ccc}
2.064 & 0.605 & -1.958 \\
0.605 & 1.207 & 0.276 \\
-1.958 & 0.276 & 3.142
\end{array}\right), \\
& K_{A 123 P 4}=k_{a c t}\left(\begin{array}{ccc}
2.191 & 0.542 & -2.220 \\
0.542 & 1.044 & 0.296 \\
-2.220 & 0.296 & 3.660
\end{array}\right), \\
& K_{A 123 P 5}=k_{a c t}\left(\begin{array}{ccc}
2.377 & 0.478 & -2.611 \\
0.478 & 0.914 & 0.334 \\
-2.611 & 0.334 & 4.446
\end{array}\right)
\end{aligned}
$$

The above stiffness matrices are symmetrical matrices where the element of row 1 and column 1 denotes the actuated stiffness $k_{\omega}$ around axis $z$ and under the external moment load, the element of row 2 and column 2 denotes the actuated stiffness $k_{x}$ along axis $x$ and under the external force along axis $x$, and the element of row 3 and column 3 denotes the actuated stiffness $k_{y}$ along axis $y$ and under the external force along axis $y$.

When joints $J_{1}, J_{2}$ and $J_{6}$ are taken as the active joints, the calculation of the Jacobian matrix is much more complicated. The joint velocities are expressed as the velocity of point $P$ in both left and right limbs

$\left(\begin{array}{l}\omega_{J_{1}} \\ \omega_{J_{2}} \\ \omega_{J_{3}}\end{array}\right)=\left(\begin{array}{ccc}1 & 1 & 1 \\ -\left(J_{1} P\right)_{y} & -\left(J_{2} P\right)_{y} & -\left(J_{3} P\right)_{y} \\ \left(J_{1} P\right)_{x} & \left(J_{2} P\right)_{x} & \left(J_{3} P\right)_{x}\end{array}\right)^{-1}\left(\begin{array}{c}\omega_{P} \\ v_{P x} \\ v_{P y}\end{array}\right)$ 


$$
\left(\begin{array}{l}
\omega_{J_{4}} \\
\omega_{J_{5}} \\
\omega_{J_{6}}
\end{array}\right)=\left(\begin{array}{ccc}
1 & 1 & 1 \\
-\left(J_{4} P\right)_{y} & -\left(J_{5} P\right)_{y} & -\left(J_{6} P\right)_{y} \\
\left(J_{4} P\right)_{x} & \left(J_{5} P\right)_{x} & \left(J_{6} P\right)_{x}
\end{array}\right)^{-1}\left(\begin{array}{l}
\omega_{P} \\
v_{P x} \\
v_{P y}
\end{array}\right)
$$

The first and second rows of the square matrix in Eqn. (24) and the third row of the square matrix in Eqn. (25) are extracted and recombined into a new matrix that is the Jacobian matrix $J_{126}$. Hence, the Jacobian matrices of the manipulator at the five points along the line $P_{1} P_{5}$ are

$$
\begin{aligned}
J_{126 P 1} & =\left(\begin{array}{ccc}
-1.193 & -1.58 & 0.943 \\
-1.1 & -0.566 & 0 \\
-0.377 & 0.192 & 0.377
\end{array}\right), \\
J_{126 P 2} & =\left(\begin{array}{ccc}
-1.158 & -1.515 & 0.908 \\
-1.2 & -0.618 & 0 \\
-0.363 & 0.18 & 0.363
\end{array}\right), \\
J_{126 P 3} & =\left(\begin{array}{ccc}
-1.086 & -1.423 & 0.836 \\
-1.3 & -0.669 & 0 \\
-0.334 & 0.173 & 0.334
\end{array}\right), \\
J_{126 P 4} & =\left(\begin{array}{ccc}
-0.967 & -1.299 & 0.717 \\
-1.4 & -0.722 & 0 \\
-0.287 & 0.172 & 0.287
\end{array}\right), \\
J_{126 P 5} & =\left(\begin{array}{ccc}
-0.775 & -1.126 & 0.525 \\
-1.5 & -0.774 & 0 \\
-0.21 & 0.182 & 0.21
\end{array}\right)
\end{aligned}
$$

When joints $J_{1}, J_{2}$ and $J_{6}$ are taken as the active joints, the matrices of the actuated stiffness at the five points are also solved according to Eqn. (10)

$$
K_{A 126 P 1}=k_{\text {act }}\left(\begin{array}{ccc}
0.620 & -0.865 & -0.144 \\
-0.865 & 2.014 & -0.567 \\
-0.144 & -0.567 & 3.873
\end{array}\right) \text {, }
$$

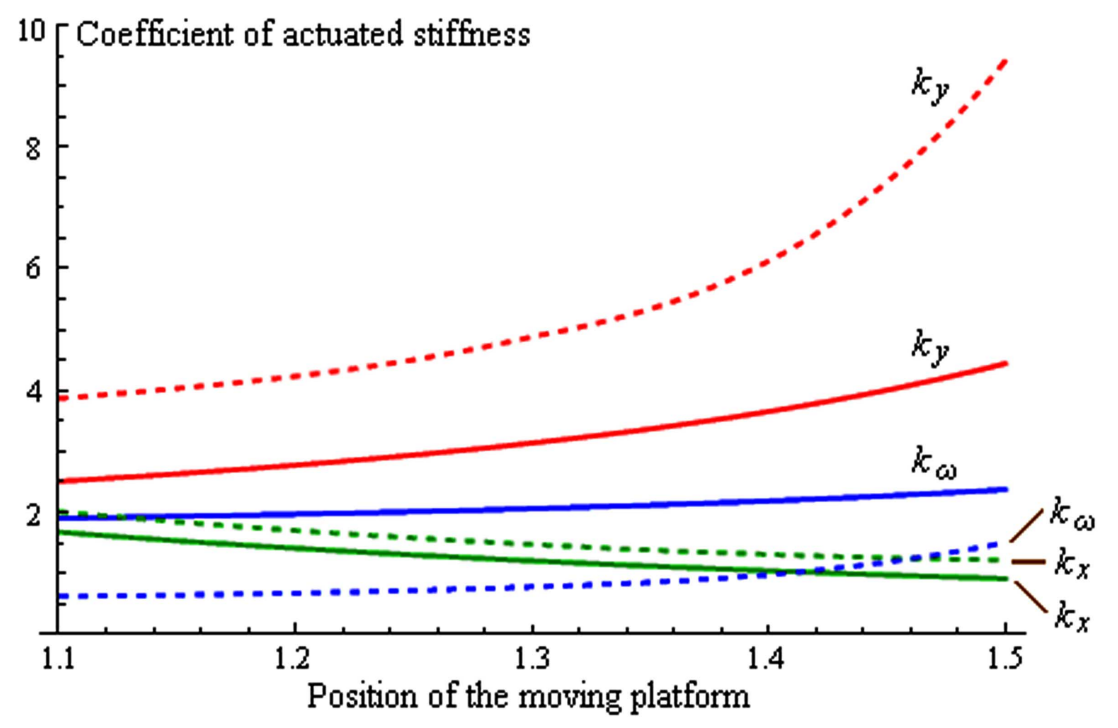

Fig. (10). Comparison of the actuated stiffness between two allocation schemes.

$$
\begin{aligned}
& K_{A 126 P 2}=k_{a c t}\left(\begin{array}{ccc}
0.678 & -0.834 & -0.179 \\
-0.834 & 1.707 & -0.526 \\
-0.179 & -0.526 & 4.237
\end{array}\right), \\
& K_{A 126 P 3}=k_{a c t}\left(\begin{array}{ccc}
0.782 & -0.840 & -0.165 \\
-0.840 & 1.478 & -0.546 \\
-0.165 & -0.546 & 4.888
\end{array}\right), \\
& K_{A 126 P 4}=k_{a c t}\left(\begin{array}{ccc}
0.981 & -0.904 & -0.018 \\
-0.904 & 1.312 & -0.667 \\
-0.018 & -0.667 & 6.1297
\end{array}\right), \\
& K_{A 126 P 5}=k_{\text {act }}\left(\begin{array}{ccc}
1.507 & -1.123 & 0.766 \\
-1.123 & 1.221 & -1.122 \\
0.766 & -1.122 & 9.421
\end{array}\right)
\end{aligned}
$$

The comparison of the coefficients of actuated stiffness between the two allocation schemes is shown in Fig. (10), where the solid curves denote the scheme in which joints $J_{1}$, $J_{2}$ and $J_{3}$ are taken as the active joints, the dashed curves denote the scheme in which joints $J_{1}, J_{2}$ and $J_{6}$ are taken as the active joints, and the red, green, and blue curves represent the actuated stiffness $k_{y}, k_{x}$ and $k_{\omega}$ respectively.

Secondly, we solve the structural stiffness, and only concern about the stiffness component in the direction of axis $y$ for the convenient discussion. The bending deformation is regarded as the main factor to the structural flexibility, and other secondary deformations and gravity forces of links are ignored. When joints $J_{1}, J_{2}$ and $J_{3}$ are taken as the active joints, the results of $\int_{0}^{l} \tilde{M}^{2} d s$ in Eqn. (14) at the five points are shown in Table 3.

Hence, the structural stiffness at the five points are calculated according to Eqn. (14):

$$
\begin{aligned}
& K_{S 123 P 1}=0.662 E I_{z}, K_{S 123 P 2}=0.694 E I_{z}, K_{S 123 P 3}=0.733 E I_{z}, \\
& K_{S 123 P 4}=0.782 E I_{z}, K_{S 123 P 5}=0.846 E I_{z}
\end{aligned}
$$


Table 3. The Results of $\int_{0}^{l} \tilde{M}^{2} d s$ at the Five Points when Joints $\boldsymbol{J}_{\mathbf{1}}, \boldsymbol{J}_{\mathbf{2}}$ and $\boldsymbol{J}_{\mathbf{3}}$ are taken as Active Joints

\begin{tabular}{|c|c|c|c|c|c|c|c|}
\hline$\overbrace{\text { Values }\left(\mathrm{m}^{3}\right)}^{\text {Links }}$ & $J_{1} J_{2}$ & $J_{2} J_{3}$ & $J_{3} P$ & $J_{4} P$ & $J_{4} J_{5}$ & $J_{5} J_{6}$ & Total \\
\hline$P_{1}$ & 0.708 & 0.716 & 0.021 & 0.064 & 0 & 0 & 1.510 \\
\hline$P_{2}$ & 0.675 & 0.681 & 0.021 & 0.064 & 0 & 0 & 1.441 \\
\hline$P_{3}$ & 0.638 & 0.641 & 0.021 & 0.064 & 0 & 0 & 1.364 \\
\hline$P_{5}$ & 0.550 & 0.546 & 0.021 & 0.064 & 0 & 0 & 1.182 \\
\hline
\end{tabular}

Table 4. The Results of $\int_{0}^{l} \tilde{M}^{2} d s$ at the Five Points when Joints $\boldsymbol{J}_{\mathbf{1}}, \boldsymbol{J}_{\mathbf{2}}$ and $\boldsymbol{J}_{\mathbf{6}}$ are taken as Active Joints

\begin{tabular}{|c|c|c|c|c|c|c|c|}
\hline & & & & & & \\
\hline
\end{tabular}

When joints $J_{1}, J_{2}$ and $J_{6}$ are taken as the active joints, the results of $\int_{0}^{l} \tilde{M}^{2} d s$ in Eqn. (14) at the five points are shown in Table 4.

Hence, the structural stiffness at the five points are also calculated according to Eqn. (14):

$$
\begin{aligned}
& K_{S 126 P 1}=5.352 E I_{z}, K_{S 126 P 2}=5.768 E I_{z}, K_{S 126 P 3}=6.700 E I_{z}, \\
& K_{S 126 P 4}=8.665 E I_{z}, K_{S 126 P 5}=13.467 E I_{z}
\end{aligned}
$$

The comparison of the coefficients of structural stiffness between the two allocation schemes is shown in Fig. (11), where the solid curves denote the scheme in which joints $J_{1}$, $J_{2}$ and $J_{3}$ are taken as the active joints, and the dashed curves denote the scheme in which joints $J_{1}, J_{2}$ and $J_{6}$ are taken as the active joints.

The mechanism stiffness is the linear superimposition of the actuated stiffness and the structural stiffness. From Figs. (6) and (7) can we see that, if the component of mechanism stiffness in the direction of axis $y$ is the chiefly concerned stiffness, the allocation scheme where joints $J_{1}, J_{2}$ and $J_{6}$ are taken as the active joints is much better than the other allocation scheme where joints $J_{1}, J_{2}$ and $J_{3}$ are taken as the active joints. Note that in the comparison of stiffness, the precise values of the physical parameters of links such as $E$ and $I_{z}$ are not required.

For the analysis of the actuated stiffness, the computational difficulty is calculation of the Jacobian matrix that depends on the allocation scheme of actuators and the pose of the manipulator. For the analysis of the structural stiffness, the computational difficulty is calculation of the deformations of links through the integral of internal forces that depend on the external loads and the pose of the manipulator. Although the numerical computation by hand for the stiffness analysis is complex and time-consuming, the analysis process can be coded as the computer program and in this way the mechanism stiffness can be obtained handily according to the input parameters that are the position and orientation of the moving platform.

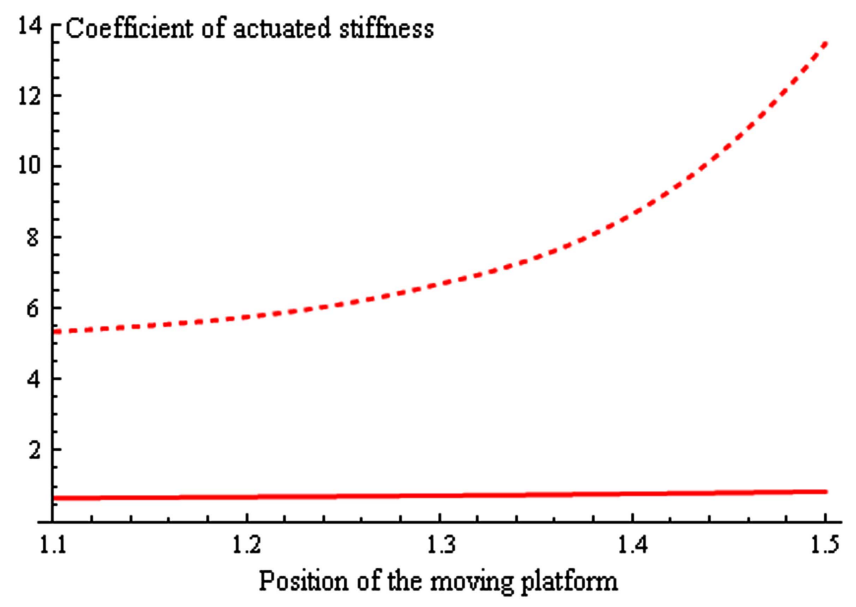

Fig. (11). Comparison of the structural stiffness between two allocation schemes. 
It is noted that only two schemes of allocation are analyzed in this paper for the convenient discussion. In one scheme, the three actuators are allocated at joints $J_{1}, J_{2}$ and $J_{3}$; in the other scheme, the three actuators are allocated at joints $J_{1}, J_{2}$ and $J_{6}$, which has been proved to be the better allocation scheme. In fact, there may be better scheme in all the 20 types of allocation. However, as discussed in Section 4 , there are many design indices to be considered at the stage of mechanism design, and even some of these indices are contradictory to each other. Therefore it needs to make a compromise between the design indices except for the mechanism stiffness, such as machine weight, total moment of inertia and manufacturing cost. The algorithm for the optimization of allocation schemes is not studied in this paper, and may be researched in the future.

\section{CONCLUSIONS}

(1) The stiffness analysis that is usually investigated at the stage of structure design in the traditional process of mechanical design is considered in anticipation at the stage of mechanism design in the proposed approach. The stiffness of the moving platform affected by the allocation of actuators is analyzed through the simplified mechanism model without the detailed geometric shapes or physical parameters of link parts, which is able to guild the optimal selection of an allocation scheme of actuators at the early stage of the design process.

(2) The mechanism stiffness of a parallel manipulator is classified into the actuated stiffness and the structural stiffness. For the actuated stiffness, the proposed approach is suitable for all types of actuators; for the structural stiffness, the proposed approach takes account of all kinds of internal forces such as axial force, shearing force, bending moment, and torque. It can be seen that the allocation scheme of actuators plays an important role in the contribution to the stiffness of a parallel mechanism.

(3) The actuated stiffness is derived from the principle of virtual displacement and Jacobian matrix, and it is presented as a stiffness matrix which contains all the stiffness components and maps the stiffness of each actuator into the stiffness of the moving platform.

(4) The structural stiffness is analyzed based on the principle of virtual force. A parallel mechanism is converted into a stationary structure when all active joints are frozen. The stationary structure is regarded as the combination of the basic links which are categorized according to their geometric shapes, join types, and load conditions. The structural stiffness is obtained at the stage of mechanism design where only the simplified link models are used but the detailed geometric or physical parameters of each link structure are not needed.

\section{CONFLICT OF INTEREST}

None declared.

\section{ACKNOWLEDGEMENTS}

This work was partially supported by the National Natural Science Foundation of China under Grant 51005199 and the China Postdoctoral Science Foundation under Grants 20100471717 and 201104719.

\section{REFERENCES}

[1] G. Carbone, and M. Ceccarelli, "A stiffness analysis for a hybrid parallel-serial manipulator”, Robotica, vol. 22, pp. 567-576, 2004.

[2] G. Carbone and M. Ceccarelli, "Comparison of indices for stiffness performance evaluation”, Front. Mech. Eng., vol. 5, pp. 270-278, 2010 .

[3] W. Yoon, T. Suehiro, Y. Tsumaki, and M. Uchiyama, "Stiffness analysis and design of a compact modified delta parallel mechanism", Robotica, vol. 22, pp. 463-475, 2004.

[4] Q. Xu, and Y. Li, "An investigation on mobility and stiffness of a 3-DOF translational parallel manipulator via screw theory", Robot Comput.-Integr. Manuf., vol. 24, pp. 402-414, 2008.

[5] T. Bonnemains, H. Chanal, B. Bouzgarrou, and P. Ray, "Stiffness computation and identification of parallel kinematic machine tools", J. Manuf. Sci. E.-T. ASME, vol. 131, pp. 041013.1041013.7, 2009.

[6] Y. Lu, B. Hu, and J. Yu, "Analyses of the stiffness and elastic deformation of a 2(3-SPR) serial-parallel manipulator", Proc. Inst. Mech. Eng. K-J. Multi-body Dyn., vol. 223, pp. 189-198, 2009.

[7] A. Pashkevich, D. Chablat, and P. Wenger, "Stiffness analysis of overconstrained parallel manipulators", Mech. Mach. Theory, vol. 44, pp. 966-982, 2009.

[8] J. Wu, J. Wang, L. Wang, T. Li, and Z. You, "Study on the stiffness of a 5-DOF hybrid machine tool with actuation redundancy", Mech. Mach. Theory, vol. 44, pp. 289-305, 2009.

[9] J. Enferadi, and A. Tootoonchi, "Accuracy and stiffness analysis of a 3-RRP spherical parallel manipulator", Robotica, vol. 29, pp. 193-209, 2011.

[10] Z. Gao, D. Zhang, X. Hu, and Y. Ge, "Design, analysis, and stiffness optimization of a three degree of freedom parallel manipulator", Robotica, vol. 28, pp. 349-357, 2010.

[11] C. Pinto, J. Corral, O. Altuzarra, and A. Hernández, "A methodology for static stiffness mapping in lower mobility parallel manipulators with decoupled motions", Robotica, vol. 28, pp. 719$735,2010$.

[12] A. Vaidya, and P. Padole, "A performance evaluation of four bar mechanism considering flexibility of links and joints stiffness", Open Mech. Eng. J., vol. 4, pp. 16-28, 2010.

[13] J. Zhao, F. Chu, and Z. Feng, "Symmetrical characteristics of the workspace for spatial parallel mechanisms with symmetric structure", Mech. Mach. Theory, vol. 43, pp. 427-444, 2008. 